\title{
Altered NCF2, NOX2 mRNA Expression Levels in Peripheral Blood Mononuclear Cells of Pulmonary Tuberculosis Patients
}

\author{
Hong-Miao Li ${ }^{1,2}$ \\ Qian Huang' \\ Fen Tang $^{2}$ \\ Tian-Ping Zhang ${ }^{3}$ \\ 'Department of Epidemiology and \\ Biostatistics, School of Public Health, \\ Anhui Medical University, Hefei, 230032, \\ Anhui, People's Republic of China; ${ }^{2}$ Anhui \\ Chest Hospital (Anhui Provincial TB \\ Institute), Hefei, 230022, Anhui, People's \\ Republic of China; ${ }^{3}$ The First Affiliated \\ Hospital of USTC, Division of Life \\ Sciences and Medicine, University of \\ Science and Technology of China, Hefei, \\ Anhui, 23000I, People's Republic of \\ China
}

\begin{abstract}
Background: Reactive oxygen species (ROS) generated by NADPH oxidase has a pivotal role in the nonspecific innate immune response to invading microorganisms including M. tuberculosis (MTB). NCF2 and NOX2 were considered as important functional subunits of NADPH oxidase complex; hence, this study aimed to evaluate the NCF2, NOX2 mRNA expressions in PBMC of pulmonary tuberculosis (PTB) patients.
\end{abstract}

Methods: A total of 79 PTB patients and 73 controls were included in our study. Quantitative real-time reverse transcription polymerase chain reaction (qRT-PCR) was used to measure the NCF2, NOX2 mRNA levels, and receiver operating characteristic (ROC) curve analysis was performed to assess the diagnostic value of NCF2, NOX2 in PTB patients.

Results: When compared to controls, the NCF2, NOX2 mRNA levels were significantly increased in PBMC from PTB patients $(P<0.001)$. However, the NCF2, NOX2 mRNA levels were not associated with major clinical and laboratory data of PTB patients. Area under curve (AUC) of ROC curve analysis for NCF2 and NOX2 were 0.686 (95\% CI: 0.601, $0.770)$ and 0.705 (95\% CI: $0.623,0.787)$, respectively.

Conclusion: Altered NCF2, NOX2 mRNA levels in PTB patients implied that these genes might play roles in PTB, and their expression levels might be potential biomarkers for the diagnosis of PTB.

Keywords: pulmonary tuberculosis, NADPH oxidase complex, NCF2, NOX2, peripheral blood mononuclear cells

\section{Introduction}

Tuberculosis (TB) remains the huge challenge to people's health and the leading cause of death from a single infectious disease with an estimated 1.5 million people deaths worldwide in 2018. ${ }^{1}$ M. tuberculosis (MTB) is the pathogen of pulmonary TB (PTB), which is most commonly spread person-to-person through cough aerosols because MTB has an ability to disrupt and evade host immune defenses, leading to its remarkable global spread. ${ }^{2}$ As an intracellular pathogen, MTB could take advantage of macrophage phagocytosis to assist in the occurrence of infection and protected dissemination to lymphoid tissue and other organs. ${ }^{3,4}$ However, macrophages could also eliminate MTB by activating pathogen recognition receptors with a lysosomal-trafficking pathway, and most people infected with MTB do not eventually develop active PTB. ${ }^{5}$ Studies revealed that reactive oxygen species (ROS) directly killed MTB, which contributed to the lysosomal-trafficking
Correspondence: Tian-Ping Zhang

The First Affiliated Hospital of University of Science and Technology of China, 17 Lujiang Road, Hefei, Anhui, 23000I, People's Republic of China

Email zhangtianping@ustc.edu.cn 
pathway to clear this pathogen, ${ }^{6,7}$ and indirectly eliminated MTB through multifarious non-oxidative mechanisms including participating autophagy and formation of neutrophil extracellular traps. ${ }^{8}$ Therefore, ROS reduction might lead to infection occurrence, and ROS regulators might give rise to immune dysfunction.

Among the various mechanisms of ROS production, the assembled nicotinamide adenine dinucleotide phosphate (NADPH) oxidase complex played a crucial role. Combined with the fact that ROS produced by NADPH oxidase complex was thought to play a pivotal role during the entire innate immune defenses during the respiratory burst, ${ }^{9}$ the phagocyte NADPH oxidase dysfunction resulted in a primary immunodeficiency, chronic granulomatous disease (CGD), which exhibited a higher risk of clinical PTB and Bacillus Calmette-Guerin complications. ${ }^{10,11}$ Therefore, the study of the relationship between the NADPH oxidase functions and PTB development had an important theoretical basis.

NADPH oxidase was a multi-subunit enzyme, comprising gp91phox, p22phox, p47phox, p67phox, p40phox, encoded by $C Y B B, C Y B A, N C F 1, N C F 2, N C F 4$ genes, respectively. ${ }^{12,13} \mathrm{NCF} 2$, also known as p67phox, was an essential component for phagocyte ROS generation. NOX2, also named as gp91phox, was another important functional subunit of NADPH oxidase complex and stored in the intracellular granule and activated by other subunits under the stimulation of phagocytosis to produce $\mathrm{O}_{2}{ }^{-14}$ At present, many studies had been performed to explore the association of polymorphisms in genes of NADPH oxidase complex with multiple diseases including systemic lupus erythematosus, Crohn's disease and TB, ${ }^{15-19}$ and the results demonstrated that $N C F 2, C Y B B$ gene polymorphisms were significantly associated with TB susceptibility. However, to our knowledge, few studies have assessed the mRNA levels of NCF2, NOX2 in peripheral blood mononuclear cells (PBMCs) from PTB patients. To further explore the role of NCF2, NOX2 in PTB development, we conducted the present study to investigate the mRNA levels of NCF2, NOX2 in PBMCs of PTB patients. In addition, ROC curves were drawn to evaluate the role of NCF2, NOX2 mRNA levels in PTB diagnosis.

\section{Materials and Methods}

\section{PTB Patients and Healthy Controls}

In the present study, the unrelated ethnic Han Chinese were recruited from June to October 2019 as subjects.
Among them, PTB patients came from the Department of Tuberculosis at Anhui Chest Hospital (Anhui Provincial TB Institute). The PTB patients were diagnosed by clinical physicians according to the following criteria: suspicious clinical symptoms, chest radiography, sputum and/or bronchoalveolar lavage fluid MTB culture, microscopy for acid fast bacilli, and effect of anti-TB treatment. The PTB patients with cancer, hepatitis, immune-compromised conditions and HIV infection were excluded in this study. The healthy volunteers, with normal chest radiographic results, no history of $\mathrm{TB}$, were recruited from the same region as healthy controls.

The clinical features of PTB patients were collected from the medical records, such as drug resistance, chest radiography, and sputum smear. In addition, several laboratory data, including total bilirubin (TBIL), aspartate aminotransferase (AST), alanine aminotransferase (ALT), and erythrocyte sedimentation rate (ESR), were also collected. The medical ethics committee of Anhui Chest Hospital (K2020-005) approved this research in accordance with Helsinki Convention, and informed consents from all subjects were obtained.

\section{Quantitative Real-Time Reverse Transcription Polymerase Chain Reaction (qRT-PCR)}

We extracted $5 \mathrm{~mL}$ peripheral blood samples from each subject using EDTA anticoagulant tube and separated PBMC from peripheral blood using Ficoll-Hypaque density gradient centrifugation. Then, the total RNA in each PBMC sample was extracted using TRIzol Reagent (Invitrogen, Carlsbad, CA, USA), and the RNA concentration was determined using NanoDrop 2000 spectrophotometer (Thermo Scientific, USA). Finally, total RNA, which was treated by DNase, was reverse-transcribed into cDNA by PrimeScriptTM RT reagent Kit (Takara Bio Inc., Japan). In this study, the maximum permissible total RNA content was $1 \mu \mathrm{g}$ in the $20 \mu \mathrm{L}$ reverse transcription reaction system; hence, the required RNA content of each sample was determined according to the RNA concentration.

In order to detect mRNA expression levels of NCF2 (sense primer: 5'-CCCCTTTCAG AAGACAGCAT-3', antisense primer: 5-CAGGTCCTCTGGTTGGGTAG-3'), NOX2 (sense primer: 5'- CAAGATGCGTGGAAACTACC-3', antisense primer: 5'- TTGAGAAT GGATGCGAAGG -3'), we performed PCR experiment with SYBR Green (SYBR Premix 
Ex Taq II, Takara Bio Inc, Japan) on QuantStudio 12K Flex Real-Time PCR System (Applied Biosystems, Foster City, CA, USA). The relative mRNA expression levels of NCF2, NOX2 were calculated using $2^{-\Delta \Delta C t}$ normalized to housekeeping gene $\beta$-actin (sense primer: 5'- CACGAAACTAC CTTCAACTCC-3', antisense primer: 5'-CATACTCCTG CTT GCTGATC- $3^{\prime}$ ) in the same sample. ${ }^{20}$ In this study, the individual with both PCR success for NCF2, NOX2 was included for final analysis.

\section{Statistical Analysis}

The NCF2, NOX2 mRNA expression levels were shown as median value and interquartile range. Nonparametric Mann-Whitney $U$-test was used to compare the mRNA expression levels of $\mathrm{NCF} 2$, NOX2 between the two groups. The association between NCF2, NOX2 mRNA expression levels and some clinical features in PTB patients was also measured by Mann-Whitney $U$-test, and the correlation between NCF2, NOX2 mRNA levels and laboratory indexes in PTB patients was analyzed by Spearman rank correlation coefficient. The value of NCF2, NOX2 as a potential diagnostic marker for PTB was assessed with receiver operating characteristic (ROC) analysis and area under curve (AUC). SPSS statistics version 23.0 was used for the above statistical analyses, and the level of statistical significance was set as $P$ value $<0.05$.

\section{Results}

\section{Comparison of NCF2, NOX2 mRNA Levels Between PTB Patients and Controls}

The PTB group, with a mean age of $45.16 \pm 17.48$ years, consisted of 53 men and 26 women. In healthy controls, there were 54 men and 19 women, and the average age was $44.36 \pm 9.86$ years. PTB patients and healthy controls were both matched in gender and age. The NCF2, NOX2 mRNA levels in PBMCs from PTB patients and healthy controls were shown in Figures 1 and 2. The NCF2 mRNA level was significantly increased in PTB patients [1.350 $(0.723,2.362)]$ when compared to healthy controls $[0.889$ $(0.540,1.274)](P<0.001)$. Similarly, the NOX2 mRNA level was also higher in PTB patients $[1.347(0.812$, 2.098)] than that in healthy controls $[0.803(0.443$, 1.274)] $(P<0.001)$.

$$
P<0.001
$$

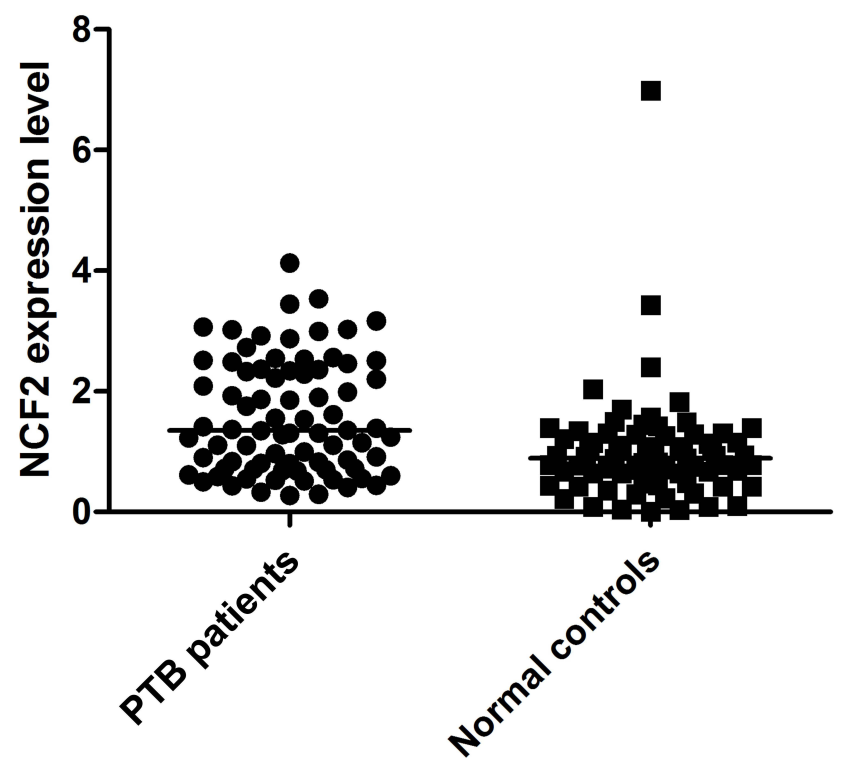

Figure I The NCF2 mRNA level in PBMCs from PTB patients and healthy controls.

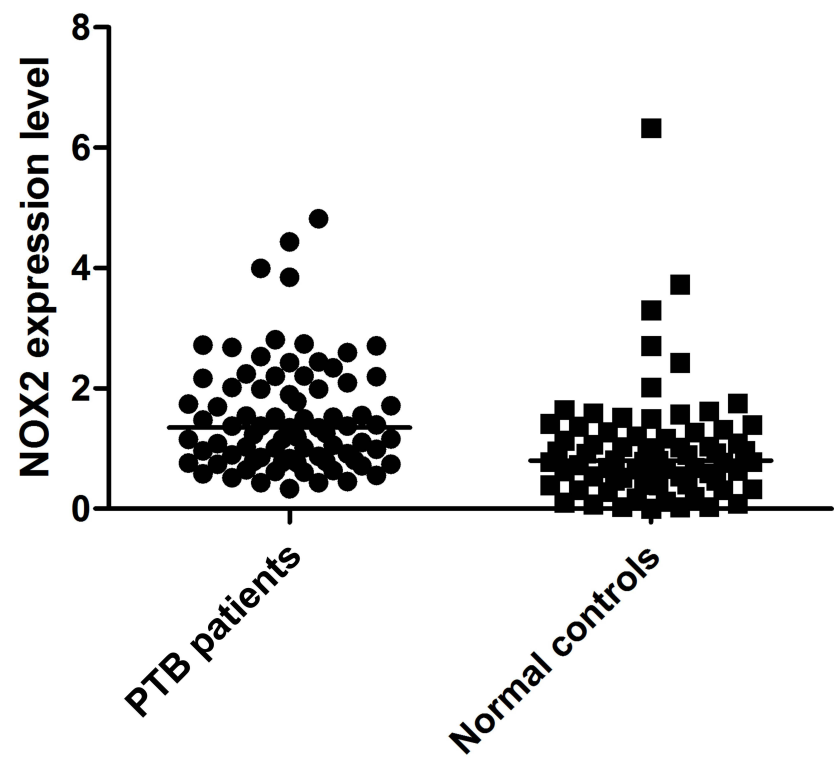

Figure 2 The NOX2 mRNA level in PBMCs from PTB patients and healthy controls.

\section{Correlations of NCF2, NOX2 mRNA}

\section{Levels with Clinical and Laboratory Data} of PTB Patients

There was no significant difference regarding NCF2, NOX2 mRNA expression levels between treatment-naive 
Table I The Correlation Between NCF2, NOX2 Expression Level and Some Clinical Features in PTB Patients

\begin{tabular}{|c|c|c|c|c|c|c|}
\hline Group & \pm & Number & NCF2 Level & $P$ value & NOX2 Level & $P$ value \\
\hline \multirow[t]{2}{*}{ Treatment-naive patients } & + & 53 & $1.311(0.715,2.417)$ & 0.900 & $1.376(0.854,2.133)$ & 0.669 \\
\hline & - & 26 & $\mathrm{I} .404(0.752,2.338)$ & & $1.216(0.785,2.042)$ & \\
\hline \multirow[t]{2}{*}{ Drug-resistant patients } & + & 17 & I.I49 $(0.564,2.367)$ & 0.311 & $1.156(0.619,2.218)$ & 0.228 \\
\hline & - & 62 & I.353 $(0.782,2.364)$ & & $1.363(0.892,2.116)$ & \\
\hline \multirow[t]{2}{*}{ Liver injury } & + & 9 & $0.996(0.7 I I, 2.652)$ & 0.793 & $1.347(0.865,1.860)$ & 0.865 \\
\hline & - & 70 & $1.362(0.719,2.364)$ & & $1.303(0.806,2.122)$ & \\
\hline \multirow[t]{2}{*}{ Diabetes } & + & 16 & $\mathrm{I} .048(0.596,2.330)$ & 0.479 & $1.206(0.785,1.87 \mathrm{I})$ & 0.457 \\
\hline & - & 63 & $1.355(0.724,2.370)$ & & $1.350(0.856,2.168)$ & \\
\hline \multirow[t]{2}{*}{ Sputum smear-positive ${ }^{a}$} & + & 31 & $1.419(0.602,2.492)$ & 0.964 & 1.376 (0.79I, 2.209) & 0.956 \\
\hline & - & 42 & $\mathrm{I} .334(0.782,2.250)$ & & $1.349(0.845,2.018)$ & \\
\hline \multirow[t]{2}{*}{ Unilateral tuberculosis foci } & + & 21 & $\mathrm{I} .149(0.82 \mathrm{I}, \mathrm{I} .394)$ & 0.340 & I.I05 (0.790, I.378) & 0.176 \\
\hline & - & 58 & I.586 $(0.702,2.47 \mathrm{I})$ & & $1.489(0.827,2.204)$ & \\
\hline \multirow[t]{2}{*}{ Pulmonary cavity } & + & 27 & $1.616(0.549,2.520)$ & 0.756 & $1.235(0.776,2.595)$ & 0.556 \\
\hline & - & 52 & $1.264(0.805,2.273)$ & & I.349 (0.823, I.87I) & \\
\hline
\end{tabular}

Notes: \pm : With/without; median (interquartile range); ${ }^{\text {a }}$ part of the study subjects of data missing.

PTB patients and treatment-experienced PTB patients, as well as PTB patients with and without drug resistance (Table 1). Our results also suggested that the NCF2, NOX2 mRNA expression levels were not associated with liver injury, diabetes, sputum smear result, tuberculosis foci, and pulmonary cavities in PTB patients (all $P>0.05)$.

The correlations of NCF2, NOX2 mRNA levels with ESR, TBIL, ALT, and AST levels of PTB patients were also analyzed, and the result is summarized in Table 2. However, no significant correlation was found (all $P>0.05)$.

Table 2 Correlation Between NCF2, NOX2 mRNA Levels and ESR, TBIL, ALT, AST of PTB Patients

\begin{tabular}{|l|c|c|c|c|}
\hline \multirow{2}{*}{ Clinical Parameters } & \multicolumn{2}{|c|}{ NCF2 Level } & \multicolumn{2}{c|}{ NOX2 Level } \\
\cline { 2 - 5 } & $\boldsymbol{r}_{\boldsymbol{s}}$ & $\boldsymbol{P}$ value & $\boldsymbol{r}_{\boldsymbol{s}}$ & $\boldsymbol{P}$ value \\
\hline ESR & 0.050 & 0.684 & 0.117 & 0.338 \\
TBIL & -0.121 & 0.290 & -0.101 & 0.375 \\
ALT & -0.144 & 0.205 & -0.146 & 0.198 \\
AST & -0.059 & 0.606 & -0.025 & 0.827 \\
\hline
\end{tabular}

Abbreviations: ESR, erythrocyte sedimentation rate; TBIL, total bilirubin; AST, aspartate aminotransferase; ALT, alanine aminotransferase; rs, Spearman rank correlation coefficient.

\section{The Value of NCF2, NOX2 as Biomarkers for PTB Diagnosis}

ROC curve analysis was performed to evaluate the predictive accuracy of NCF2, NOX2 mRNA levels for the diagnostic biomarkers of PTB. We found that NCF2 might be a promising diagnostic biomarker for PTB, with an AUC of 0.686 (95\% CI: 0.601-0.770) (Figure 3). Moreover, the optimal cut-off point of NCF2 level was 1.520 , with a $41.67 \%$ sensitivity and $90.4 \%$

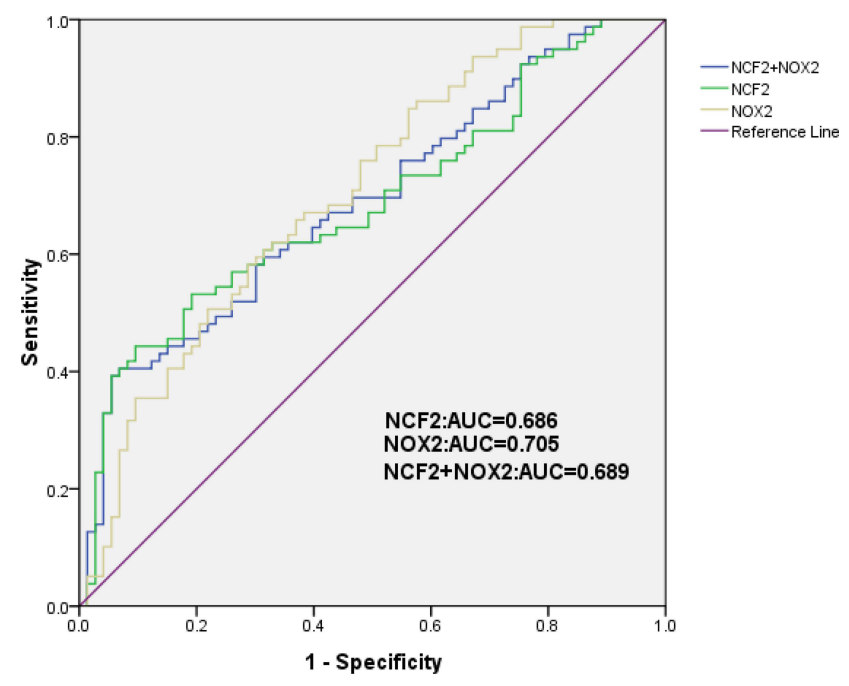

Figure 3 The diagnostic value of NCF2 and NOX2 mRNA levels in PTB. 
specificity. As for NOX2, the AUC was 0.705 (95\% CI: 0.623 0.787 ) and had a $59.5 \%$ sensitivity and $69.9 \%$ specificity with an optimal cut-off point of 1.096 (Figure 3). We also further evaluated the combined diagnostic value of NCF2 and NOX2 mRNA levels as biomarkers for PTB; however, the AUC was only 0.689 (95\% CI: 0.607, 0.773) (Figure 3).

\section{Discussion}

ROS was known to play important roles in a variety of human immune processes, including host immune defense and cell signaling, and many studies had analyzed the roles of NADPH oxidase gene polymorphisms in PTB developments. In the present study, for the first time, we detected the expression levels of NCF2, NOX2 in PBMC from PTB patients and found that their expression levels were abnormal compared with the control group.

NCF2 was reported to be involved in the higher production of the free radicals characterising B-cell activation. During activation, the three cytosolic subunits, including NCF1, NCF2, and NCF4, colocalize and translocate to the membrane, together with CYBA, CYBB, and the small GTPase, constituting an active NOX2 complex producing a stream of superoxide that was delivered to the lumen of the neutrophil phagosome. ${ }^{7,12,13}$ At present, the NCF2 genetic variants had been reported to be associated with susceptibility to multiple diseases. Cunninghame et al found that an intronic variant rs10911363 in NCF2 gene was related to SLE susceptibility in European population. ${ }^{21}$ Another study performed by Jiao et al showed that NCF2 rs10911362 variant was associated with a decreased TB susceptibility in Chinese population and was also significantly related to the decreased PTB risk. ${ }^{17}$ In this study, we explored the association between NCF2 mRNA level and PTB patients. Our results demonstrated that the NCF2 mRNA level was significantly increased in PBMC from PTB patients when compared to healthy controls. However, NCF2 mRNA level was not significantly correlated with some clinical manifestations and laboratory indicators of PTB. These results further confirmed the involvement of NCF2 in the pathogenesis of PTB. We also discussed the value of NCF2 in the early diagnosis of PTB by ROC curve analysis. The results suggested that when the optimal cut-off point was 1.520 , the specificity could reach $90.4 \%$, and NCF2 level might be used for the diagnosis of PTB patients. The significance of the combination of NCF2 with a standard screening test in PTB diagnosis should be explored in an expanded sample.
NOX2 was encoded by $C Y B B$ gene, and the possible roles of $C Y B B$ variants in ROS-associated diseases had been analyzed in CGD, diabetic nephropathy, inflammatory bowel disease, and malaria. ${ }^{8,22-24}$ Studies had shown that $C Y B B$ was located on the $\mathrm{X}$ chromosome, and $C Y B B$ mutation account for about $70 \%$ of CGD patients, which could greatly increase the risk of suffering from $\mathrm{TB}$ in high endemic areas. ${ }^{24,25}$ Therefore, the $C Y B B$ gene polymorphisms associated with CGD incidence might also be related to the risk of TB, and Liu et al found that $C Y B B$ rs5917471 was significantly associated with decreased risk of TB and PTB. ${ }^{18}$ This supported the hypothesis that NOX2 might play a role in the development of PTB. However, NOX2 mRNA expression in PBMC from PTB patients had not been studied. We found that the expression level of NOX2 mRNA in PBMC of PTB patients was elevated compared with that of healthy controls. Moreover, our results suggested that NOX2 had value as a possible, auxiliary diagnostic marker for PTB with the AUC of 0.705 (95\% CI: $0.623-$ 0.787). Our study also demonstrated that the NOX2 mRNA level in PBMC was not associated with major clinical features, laboratory indicators of PTB. This is implying that NOX2 was stable in PBMCs and could be considered as a potential diagnostic marker for PTB.

In summary, our results indicated that NCF2, NOX2 mRNA levels were significantly increased in PBMC of PTB; moreover, ROC curve analysis demonstrated that NCF2, NOX2 might serve as auxiliary biomarkers for the diagnosis of PTB. These findings suggested that these genes might play a role in PTB. However, some limitations of our study should also be acknowledged. Firstly, we did not exclude the potential effect of drug therapy on the expression level of NCF2, NOX2 mRNA in PBMC of PTB patients. Additionally, the sample size of this study might not be sufficient, and this could affect the power of this study. In addition, an experiment to explore the combination diagnostic value of NCF2, NOX2 and other diagnostic methods including T-SPOT.TB and interferon- $\gamma$ release assay in PTB is necessary in the future. Moreover, further large-scale replication studies along with functional tests are needed to verify the exact roles of NADPH oxidase complex in PTB.

\section{Data Sharing Statement}

The datasets used and/or analysed during the current study are available from the corresponding author on reasonable request. 


\section{Ethics Approval and Consent to Participate}

This study was approved by the Ethics Committee of Anhui Chest Hospital (K2020-005), and the informed consent was obtained from each participant.

\section{Acknowledgments}

We sincerely thank all the participants in this project.

\section{Author Contributions}

T-PZ primarily designed the study. H-ML primarily conducted the experiment. QH and FT primarily performed the statistical analyses. H-ML primarily drafted the manuscript. All authors made substantial contributions to conception and design, acquisition of data, or analysis and interpretation of data; took part in revising it critically for importance; and gave final approval of the version to be published.

\section{Funding}

This work was supported by National Natural ScienceFoundation of China (82003515) and Anhui Provincial Natural Science Foundation (1908085QH368).

\section{Disclosure}

The authors declare that they have no conflicts of interest.

\section{References}

1. World Health Organization. Global tuberculosis report 2019. Geneva: WHO; 2019.

2. Gagneux S, DeRiemer K, Van T, et al. Variable host-pathogen compatibility in Mycobacterium tuberculosis. Proc Natl Acad Sci US A. 2016;103(8):2869-2873. doi:10.1073/pnas.0511240103

3. Houben EN, Nguyen L, Pieters J. Interaction of pathogenic mycobacteria with the host immune system. Curr Opin Microbiol. 2006;9 (1):76-85. doi:10.1016/j.mib.2005.12.014

4. Fortune SM, Solache A, Jaeger A, et al. Mycobacterium tuberculosis inhibits macrophage response to IFN-gamma through myeloid differentiation factor 88-dependent and -independent mechanisms. J Immunol. 2004;172(10):6272-6280. doi:10.4049/jimmunol.172.10.6272

5. Köster S, Upadhyay S, Chandra P, et al. Mycobacterium tuberculosis is protected from NADPH oxidase and LC3-associated phagocytosis by the LCP protein CpsA. Proc Natl Acad Sci U S A. 2017;114(41): E8711-20. doi:10.1073/pnas.1707792114

6. Heyworth PG, Cross AR, Curnutte JT. Chronic granulomatous disease. Curr Opin Immunol. 2003;15(5):578-584. doi:10.1016/S09527915(03)00109-2

7. Martner A, Aydin E, Hellstrand K. NOX2 in autoimmunity, tumor growth and metastasis. J Pathol. 2019;247(2):151-154. doi:10.1002/ path. 5175

8. Deffert C, Cachat J, Krause K-H. Phagocyte NADPH oxidase, chronic granulomatous disease and mycobacterial infections. Cell Microbiol. 2014;16(8):1168-1178. doi:10.1111/cmi.12322
9. Liu Q, Wu S, Xue M, et al. Heterozygote advantage of the rs 3794624 polymorphism in CYBA for resistance to tuberculosis in two Chinese populations. Sci Rep. 2016;6:38213. doi:10.1038/srep38213

10. von Goessel H, Hossle JP, Seger R, Gungor T. Characterization of 17 new cases of $\mathrm{X}$-linked chronic granulomatous disease with seven novel mutations in the CYBB gene. Exp Hematol. 2006;34 (4):528-535. doi:10.1016/j.exphem.2006.01.005

11. Bustamante J, Aksu G, Vogt G, et al. BCG-osis and tuberculosis in a child with chronic granulomatous disease. $J$ Allergy Clin Immunol. 2007;120(1):32-38. doi:10.1016/j.jaci.2007.04.034

12. Nauseef WM. Assembly of the phagocyte NADPH oxidase. Histochem Cell Biol. 2004;122(4):277-291. doi:10.1007/s00418004-0679-8

13. Groemping Y, Rittinger K. Activation and assembly of the NADPH oxidase: a structural perspective. Biochem J. 2005;386:401-416. doi:10.1042/BJ20041835

14. Leto TL, Morand S, Hurt D, Ueyama T. Targeting and regulation of reactive oxygen species generation by Nox family NADPH oxidases. Antioxid Redox Signal. 2009;11(10):2607-2619. doi:10.1089/ ars.2009.2637

15. Kim-Howard X, Sun C, Molineros JE, et al. Allelic heterogeneity in NCF2 associated with systemic lupus erythematosus (SLE) susceptibility across four ethnic populations. Hum Mol Genet. 2014;23:1656-1668. doi:10.1093/hmg/ddt532

16. Denson LA, Jurickova I, Karns R, et al. Clinical and genomic correlates of neutrophil reactive oxygen species production in pediatric patients with Crohn's disease. Gastroenterology. 2018;154 (8):2097-2110. doi:10.1053/j.gastro.2018.02.016

17. Jiao L, Song J, Ding L, et al. A novel genetic variation in NCF2, the core component of NADPH oxidase, contributes to the susceptibility of tuberculosis in Western Chinese Han Population. DNA Cell Biol. 2020;39(1):57-62. doi:10.1089/dna.2019.5082

18. Liu Q, Wang J, Sandford AJ, et al. Association of CYBB polymorphisms with tuberculosis susceptibility in the Chinese Han population. Infect Genet Evol. 2015;33:169-175. doi:10.1016/j.meegid.2015.04.026

19. Tupurani MA, Padala C, Puranam K, et al. Association of CYBA gene $(-930 \mathrm{~A} / \mathrm{G}$ and $242 \mathrm{C} / \mathrm{T})$ polymorphisms with oxidative stress in breast cancer: a case-control study. PeerJ. 2018;6:e5509. doi: 10.7717 peerj.5509

20. Livak KJ, Schmittgen TD. Analysis of relative gene expression data using real-time quantitative PCR and the $2-\Delta \Delta \mathrm{CT}$ method. Methods. 2001;25:402-408. doi:10.1006/meth.2001.1262

21. Cunninghame Graham DS, Morris DL, Bhangale TR, et al. Association of NCF2, IKZF1, IRF8, IFIH1, and TYK2 with systemic lupus erythematosus. PLoS Genet. 2011;7(10):e1002341. doi:10.1371/journal.pgen.1002341

22. Uhlemann AC, Szlezák NA, Vonthein R, et al. DNA phasing by TA dinucleotide microsatellite length determines in vitro and in vivo expression of the gp91phox subunit of NADPH oxidase and mediates protection against severe malaria. $J$ Infect Dis. 2004;189 (12):2227-2234. doi:10.1086/421242

23. Monteiro MB, Patente TA, Mohammedi K, et al. Sex-specific associations of variants in regulatory regions of NADPH oxidase-2 (CYBB) and glutathione peroxidase 4 (GPX4) genes with kidney disease in type 1 diabetes. Free Radic Res. 2013;47(10):804-810. doi:10.3109/10715762.2013.828347

24. Kang EM, Marciano BE, DeRavin S, Zarember KA, Holland SM, Malech HL. Chronic granulomatous disease: overview and hematopoietic stem cell transplantation. J Allergy Clin Immunol. 2011;127 (6):1319-26 quiz 27-8. doi:10.1016/j.jaci.2011.03.028

25. Khan TA, Kalsoom K, Iqbal A, et al. A novel missense mutation in the NADPH binding domain of CYBB abolishes the NADPH oxidase activity in a male patient with increased susceptibility to infections. Microb Pathog. 2016;100:163-169. doi:10.1016/j.micpath.201 6.09 .020 


\section{Publish your work in this journal}

The International Journal of General Medicine is an international, peer-reviewed open-access journal that focuses on general and internal medicine, pathogenesis, epidemiology, diagnosis, monitoring and treatment protocols. The journal is characterized by the rapid reporting of reviews, original research and clinical studies across all disease areas. The manuscript management system is completely online and includes a very quick and fair peer-review system, which is all easy to use. Visit http://www.dovepress.com/ testimonials.php to read real quotes from published authors.

Submit your manuscript here: https://www.dovepress.com/international-journal-of-general-medicine-journal 